\title{
The Montreal Cognitive Assessment (MoCA): updated norms and psychometric insights into adaptive testing from healthy individuals in Northern Italy
}

\author{
Edoardo Nicolò Aiello ${ }^{1,2}\left(\right.$ Chiara Gramegna $^{3} \cdot$ Antonella Esposito $^{3} \cdot$ Valentina Gazzaniga $^{3} \cdot$ Stefano Zago $^{4,6}$. \\ Teresa Difonzo ${ }^{4}$. Ottavia Maddaluno ${ }^{5} \cdot$ Ildebrando Appollonio $^{1,6,7} \cdot$ Nadia Bolognini $^{3,8}$
}

Received: 15 April 2021 / Accepted: 16 July 2021 / Published online: 27 July 2021

(C) The Author(s) 2021

\begin{abstract}
Background The availability of fine-grained, culture-specific psychometric outcomes can favor the interpretation of scores of the Montreal Cognitive Assessment (MoCA), the most frequently used instrument to screen for mild cognitive dysfunctions in both instrumental and non-instrumental domains. This study thus aimed at providing: (i) updated, region-specific norms for the Italian MoCA, by also (ii) comparing them to pre-existing ones with higher geographical coverage; (iii) information on sensitivity and discriminative capability at the item level.

Methods Five hundred and seventy nine healthy individuals from Northern Italy (208 males, 371 females; age: 63.4 \pm 15 , 21-96; education: $11.3 \pm 4.6,1-25$ ) were administered the MoCA. Item Response Theory (IRT) was adopted to assess item difficulty and discrimination. Normative values were derived by means of the Equivalent Scores (ESs) method, applied to the MoCA and its sub-scales. Average ESs were also computed. Agreement with previous ESs classification was assessed via Cohen's $k$.

Results Age and education significantly predicted all MoCA measures except for Orientation, which was related to age only. No sex differences were detected when tested along with age and education. Substantial disagreements with previous ESs classifications were detected. Several items proved to be scarcely sensitive, especially the place item from Orientation and the letter detection task. Memory items showed high discriminative capability, along with certain items assessing executive functions and orientation.

Discussion Item-level information herewith provided for the Italian MoCA can help interpret its scores by Italian practitioners. Italian practitioners should consider an adaptive use of region-specific norms for the MoCA.
\end{abstract}

Keywords Montreal Cognitive Assessment $\cdot$ Cognitive impairment $\cdot$ Item Response Theory $\cdot$ Adaptive testing $\cdot$ Normative data $\cdot$ Cultural differences

Edoardo Nicolò Aiello and Chiara Gramegna equally contributed to the work.

Edoardo Nicolò Aiello

e.aiello5@campus.unimib.it

1 School of Medicine and Surgery, University of MilanoBicocca, Monza, Italy

2 PhD Program in Neuroscience, University of Milano-Bicocca, Monza, Italy

3 Department of Psychology, and NeuroMi - Milan Center for Neuroscience, University of Milano Bicocca, Milan, Italy

4 Fondazione IRCCS Ca' Granda Ospedale Maggiore Policlinico, University of Milan, Milan, Italy
5 Department of Psychology, Sapienza University of Rome, Rome, Italy

6 Milan Center for Neuroscience (NeuroMI), Milan, Italy

7 Neurology Section, School of Medicine and Surgery, University of Milano-Bicocca, Monza, Italy

8 Laboratory of Neuropsychology, IRCCS Istituto Auxologico Italiano, Milan, Italy 


\section{Introduction}

Cognitive screening/first-level tests allow an estimate of global efficiency/functioning by adequately balancing between informativity and practicality of usage [1]. Compared to screening tests for dementia [2], those aimed at detecting mild-to-moderate cognitive impairment [3] may be harder for practitioners to interpret because of (a) the magnitude of the target construct (i.e., the deficit) being less obvious and (b) the amount of information provided by the test being limited [4]. Fine-grained, adaptive psychometric approaches can thus help solve interpretation issues to facilitate diagnostic processes by magnifying informativity $[5,6]$.

The Montreal Cognitive Assessment (MoCA) [7] is one of the most widespread and psychometrically robust screening tools for cognitive impairments of graded severity [8]. The MoCA is a rapid (5-10') screening test which evaluates both non-instrumental (executive functioning, attention) and instrumental (language, memory, visuospatial abilities, orientation) domains.

In Italy, the MoCA has been adapted and standardized - and both its statistical properties and clinical usability thoroughly examined [9-12].

Psychometric investigations on the MoCA have been carried out both at the sub-test and the single-item levels $[13,14]$. A widespread approach that allows a flexible use of cognitive screening tests [15] is to provide norms for their domain-specific sub-tests [10]. Moreover, information regarding single items can further help practitioners interpret test scores by qualitatively assigning different weights to different items [16]. To this last end, Item Response Theory (IRT) analyses [17] have been conducted on MoCA items to assess both their sensitivity and discriminative capability [18-21]. IRT-based analyses indeed proved to yield relevant insights to performance interpretations; for instance, executive- and memory-related items were often shown to be highly informative [18, 19].

Further improvements to adaptive testing may come from deriving norms that account for inter-regional socio-demographic heterogeneity [22]. Cultural differences within a same country have been indeed highlighted as a relevant confounding predictor when interpreting test scores [23].

Therefore, providing region-/culture-specific psychometric fine-grained outcomes and normative data can ameliorate I-level cognitive testing in both clinical and research contexts [24].

It is furthermore worth highlighting that rapid sociodemographic changes may pose additional challenges to practitioners when drawing up-to-date clinical inferences since norms need to be frequently renewed [25].

The present study thus aimed at: (i) providing updated, region-specific normative data for the Italian MoCA and its sub-tests; (ii) comparing existing norms for the MoCA in the Italian population to those drawn from a region-specific Italian sample; (iii) providing IRT-based information regarding sensitivity and discriminative capability of MoCA items in an Italian population sample.

\section{Methods}

\section{Participants}

Five hundred and seventy nine healthy Italian native speakers were recruited in Lombardy, Northern Italy. Exclusion criteria were: (a) a confirmed diagnosis of neurological or psychiatric disorders; (b) general medical conditions possibly affecting cognition (i.e., non-compensated and/or severe metabolic/internal morbidities and systemic/organ failures); (c) intake of psychotropic drugs. Participants suffering from well-compensated metabolic/internal conditions were included $[9,10]$. Participants had normal or correctedto-normal vision and/or hearing. Sample stratification is reported in Table 1. Data were derived from three different normative studies where the MoCA was administered cognitive screening aims; the MoCA was administered as the first test in every study, adopting the same procedure (as detailed below), the same sampling criteria (as detailed above) and geographical coverage. All of these studies were approved
Table 1 Sample stratification for age, education and sex

\begin{tabular}{|c|c|c|c|c|c|c|c|c|}
\hline \multirow{2}{*}{$\begin{array}{l}\mathrm{M} / \mathrm{F} \\
\text { Education }\end{array}$} & \multicolumn{8}{|l|}{ Age } \\
\hline & $35 \leq$ & $36-45$ & $46-55$ & $56-65$ & $66-75$ & $76-85$ & $86-95$ & $\geq 96$ \\
\hline $4 \leq$ & $0 / 0$ & $0 / 0$ & $0 / 0$ & $0 / 0$ & $0 / 0$ & $2 / 4$ & $0 / 1$ & $1 / 0$ \\
\hline $5-8$ & $0 / 1$ & $5 / 2$ & $7 / 18$ & $13 / 16$ & $14 / 41$ & $25 / 64$ & $5 / 14$ & $1 / 0$ \\
\hline $9-13$ & $6 / 4$ & $5 / 10$ & $16 / 41$ & $33 / 38$ & $13 / 7$ & $9 / 17$ & $2 / 6$ & $0 / 0$ \\
\hline $14-17$ & $1 / 6$ & $0 / 4$ & $8 / 13$ & $15 / 16$ & $3 / 2$ & $1 / 2$ & $1 / 3$ & $0 / 0$ \\
\hline $18-20$ & $3 / 2$ & $1 / 4$ & $4 / 8$ & $11 / 15$ & $1 / 2$ & $1 / 1$ & $0 / 1$ & $0 / 0$ \\
\hline$\geq 21$ & $0 / 0$ & $0 / 3$ & $0 / 3$ & $2 / 1$ & $0 / 0$ & $0 / 0$ & $0 / 1$ & $0 / 0$ \\
\hline
\end{tabular}

Cells show male/female ratio for each co-occurrence 
by the Research Evaluation Committee of the Department of Psychology of University of Milano-Bicocca on behalf of the Ethical Committee of the same Institution. Participants provided informed consent and signed a data treatment disclaimer for research purposes.

\section{Materials}

The Italian version of the MoCA was administered to all participants [26]. Items were grouped as follows: Executive Functioning (EF): Trail-Making B (TMT), phonemic fluency and verbal abstraction tasks; Attention (A): serial backward subtraction, letter detection by tapping and forward/backward digit span tasks; Language (L): confrontation naming and sentence repetition ; Visuo-spatial (VS): three-dimension cube copy and Clock Drawing task (CDT); Orientation $(\mathrm{O})$ and Memory $(\mathrm{M})$ : spatio-temporal orientation and delayed recall (DR) items, respectively $[9,10]$.

\section{Statistical analyses}

Normality checks on raw variables were performed descriptively, by evaluating skewness and kurtosis values, and graphically, by visually inspecting histograms and quantilequantile plots) [27, 28]. Between-variables associations were thus tested via either parametric (Pearson's) or non-parametric (Spearman's) techniques. Sex differences were tested via independent sample $t$ tests.

MoCA reliability was assessed via an internal consistency analysis (Cronbach's $\alpha$ ), whereas construct validity by means of a Principal Component Analysis (PCA). Single-item-level analyses were performed by applying a two-parameter logistic IRT model for dichotomous outcomes via the R package $\operatorname{ltm}$ [29]; item difficulty and discrimination were thus computed [17, 30, 31]. Higher values of both parameters correspond to higher levels of the target construct. Cognitive efficiency was regarded as the latent trait.

Regression-based norms were derived via the Equivalent Scores (ESs) method [32, 33]; outer and inner tolerance limits (oTL and iTL, respectively) as well as ESs threshold were computed. Average ESs (AESs) [34] were also calculated by averaging ESs of each sub-test to provide a standardized across-domain global index.
Agreement between the present ES classification and those from previous normative studies $[9,10]$ was tested by crossing level of abilities via Cohen's $k$.

Analyses regarding MoCA total scores were performed on the whole sample, whereas those for single sub-tests and items were conducted on $N=535$ participants only due to imputation issues.

Statistical power was computed a posteriori based on the final multiple regression model $\left(d f_{\text {numerator }}=3\right)$ [35] on MoCA total scores via the R package $p w r$ [36] - according to previous normative studies $[37,38]$ and by taking into account $\alpha=0.05$ and $f^{2}$ derived from fit measures.

Analyses were performed via SPSS 27 [39] and R 3.6.3 [40]. ES-related procedures were carried out according to guidelines reported by Aiello and Depaoli [41].

\section{Results}

Participants' demographics and MoCA scores $(M \pm S D$ and range) are reported in Table 2.

Age proved to be inversely related to both total $\left(\right.$ Spearman's $\left.r_{\mathrm{s}}(579)=-0.57 ; p<0.001\right)$ and sub-test $\left(-0.46 \leq r_{\mathrm{s}}(535) \leq-0.11 ; .014 \leq p<0.001\right)$ MoCA scores, whereas a positive association with education was found for all measures: MoCA total $\left(r_{\mathrm{s}}(579)=0.55 ; p<0.001\right)$ and sub-test $\left(0.15 \leq r_{\mathrm{s}}(535) \leq 0.53 ; p \leq .001\right)$ scores. Sex differences were detected with respect to MoCA-A $(t(441.8)=2.42 ; p=0.021$; males: $5.52 \pm .81$; females: $5.33 \pm 0.95),-\mathrm{L}(t(482.98)=2.96 ; p=0.003$; males: $4.6 \pm 0.6$; females: $4.42 \pm 0.79)$ and $-\operatorname{VS}(t(533)=2.12$; $p=.034$; males: $3.22 \pm 0.92$; females: $3.03 \pm 0.98)$ scores. Moreover, males $(24.57 \pm 3.47)$ scored slightly higher $(t(494.4)=1.96 ; p=0.05)$ than females $(23.94 \pm 4.15)$ on the MoCA-total. However, when simultaneously tested, only age and education proved to be significantly predictive of all MoCA measures (age: $|0.19| \leq \beta \leq 10.381 ; p<0.001$; education: $|0.16| \leq \beta \leq|0.42|$; $p<0.001)$; however, MoCA-O was found to be predicted by age only $(\beta=0.19 ; p<0.001)$. Achieved power was estimated at $1-\beta \approx 1$, with an effect size $f^{2}=R^{2} /\left(1-R^{2}\right)=0.45 /(1-0.45)=0.82$.

Adjustment equations and grids as well as TLs and ESs thresholds are reported in Tables 3 and 4, respectively. Since both MoCA-M TLs corresponded to negative values, the

Table 2 Participants' demographics and cognitive variables

\begin{tabular}{|c|c|c|c|c|c|c|c|c|c|}
\hline $\operatorname{Sex}(M / F)$ & Age (years) & $\begin{array}{l}\text { Education } \\
\text { (years) }\end{array}$ & $\begin{array}{l}\text { MoCA } \\
(N=579)\end{array}$ & $\begin{array}{l}\text { MoCA-VS } \\
(N=535)\end{array}$ & $\begin{array}{l}\text { MoCA-EF } \\
(N=535)\end{array}$ & $\begin{array}{l}\text { MoCA-L } \\
(N=535)\end{array}$ & $\begin{array}{l}\text { MoCA-A } \\
(N=535)\end{array}$ & $\begin{array}{l}\text { MoCA-M } \\
(N=535)\end{array}$ & $\begin{array}{l}\text { MoCA-O } \\
(N=535)\end{array}$ \\
\hline $208 / 371$ & $\begin{array}{c}63.44 \pm 15.04 \\
(21-96)\end{array}$ & $\begin{array}{l}11.27 \pm 4.6 \\
(1-25)\end{array}$ & $\begin{array}{c}24.17 \pm 3.93 \\
(8-30)\end{array}$ & $\begin{array}{c}3.1 \pm .97 \\
(0-4)\end{array}$ & $\begin{array}{l}2.94 \pm 1.12 \\
(0-4)\end{array}$ & $\begin{array}{c}4.48 \pm .73 \\
(1-5)\end{array}$ & $\begin{array}{c}5.4 \pm .91 \\
(1-6)\end{array}$ & $\begin{array}{l}2.33 \pm 1.81 \\
(0-5)\end{array}$ & $\begin{array}{l}5.9 \pm 0.5 \\
\quad(2-6)\end{array}$ \\
\hline
\end{tabular}

MoCA, Montreal Cognitive Assessment; VS, visuo-spatial; EF, executive functioning; L, language; A, attention; M, memory; O, orientation. Continuous outcomes are reported as $M \pm S D$ and range (in brackets) 
Table 3 Adjustment grids according to age and education for MoCA total and sub-test raw scores

\begin{tabular}{|c|c|c|c|c|c|c|c|c|c|c|c|c|c|c|}
\hline \multirow[t]{2}{*}{ Sub-test } & \multirow[t]{2}{*}{ Education } & \multicolumn{13}{|l|}{ Age } \\
\hline & & 35 & 40 & 45 & 50 & 55 & 60 & 65 & 70 & 75 & 80 & 85 & 90 & 95 \\
\hline \multirow[t]{7}{*}{ Total } & 5 & 0.35 & 0.52 & 0.73 & 1 & 1.34 & 1.73 & 2.2 & 2.75 & 3.38 & 4.1 & 4.92 & 5.84 & 6.86 \\
\hline & 8 & -1.22 & -1.05 & -0.83 & -0.56 & -0.23 & .17 & .64 & 1.18 & 1.81 & 2.53 & 3.35 & 4.27 & 5.3 \\
\hline & 11 & -2.28 & -2.11 & -1.89 & -1.62 & -1.29 & -0.89 & -0.43 & 0.12 & 0.75 & 1.47 & 2.29 & 3.21 & 4.24 \\
\hline & 13 & -2.84 & -2.67 & -2.45 & -2.18 & -1.85 & -1.45 & -0.98 & -0.43 & 0.2 & 0.92 & 1.73 & 2.65 & 3.68 \\
\hline & 16 & -3.53 & -3.36 & -3.14 & -2.87 & -2.54 & -2.14 & -1.67 & -1.13 & -0.5 & 0.23 & 1.04 & 1.96 & 2.99 \\
\hline & 18 & -3.92 & -3.75 & -3.53 & -3.26 & -2.93 & -2.53 & -2.07 & -1.52 & -0.89 & -0.17 & 0.65 & 1.57 & 2.6 \\
\hline & 21 & -4.43 & -4.26 & -4.05 & -3.78 & -3.45 & -3.05 & -2.58 & -2.03 & -1.4 & -0.68 & 0.14 & 1.06 & 2.08 \\
\hline \multirow[t]{7}{*}{ VS } & 5 & - & 0.06 & 0.13 & 0.2 & 0.28 & 0.37 & 0.47 & 0.57 & 0.69 & 0.81 & 0.93 & 1.07 & 1.21 \\
\hline & 8 & -3 & -0.24 & -0.18 & -0.1 & -0.02 & 0.07 & 0.17 & 0.27 & 0.38 & 0.5 & 0.63 & 0.77 & 0.91 \\
\hline & 11 & -0.51 & -0.45 & -0.38 & -0.31 & -0.23 & -0.14 & -0.04 & 0.06 & 0.18 & 0.3 & 0.42 & 0.56 & 0.7 \\
\hline & 13 & -0.61 & -0.55 & -0.49 & -0.42 & -0.33 & -0.24 & -0.15 & -0.04 & 0.07 & 0.19 & 0.32 & 0.45 & 0.6 \\
\hline & 16 & -0.75 & -0.69 & -0.62 & -0.55 & -0.47 & -0.38 & -0.28 & -0.18 & -0.07 & 0.05 & 0.18 & 0.32 & 0.46 \\
\hline & 18 & -0.82 & -0.77 & -0.7 & -0.63 & -0.54 & -0.46 & -0.36 & -0.25 & -0.14 & -0.02 & 0.11 & 0.24 & 0.39 \\
\hline & 21 & -0.92 & -0.86 & -0.8 & -0.73 & -0.64 & -0.55 & -0.46 & -0.35 & -0.24 & -0.12 & 0.01 & 0.14 & 0.29 \\
\hline \multirow[t]{7}{*}{$\mathrm{EF}$} & 5 & 0.22 & 0.26 & 0.31 & 0.38 & 0.46 & 0.56 & 0.68 & 0.82 & 0.97 & 1.15 & 1.36 & 1.59 & 1.84 \\
\hline & 8 & -0.25 & -0.21 & -0.16 & -0.09 & -0.01 & 0.09 & 0.21 & 0.35 & 0.51 & 0.69 & 0.89 & 1.12 & 1.38 \\
\hline & 11 & -0.57 & -0.53 & -0.47 & -0.41 & -0.32 & -0.22 & -0.11 & 0.03 & 0.19 & 0.37 & 0.57 & 0.8 & 1.06 \\
\hline & 13 & -0.74 & -0.69 & -0.64 & -0.57 & -0.49 & -0.39 & -0.27 & -0.14 & 0.02 & 0.2 & 0.41 & 0.64 & 0.89 \\
\hline & 16 & -0.94 & -0.9 & -0.85 & -0.78 & -0.7 & -0.6 & -0.48 & -0.34 & -0.19 & -0.01 & 0.2 & 0.43 & 0.69 \\
\hline & 18 & -1.06 & -1.02 & -0.96 & -0.9 & -0.81 & -0.71 & -0.6 & -0.46 & -0.3 & -0.12 & 0.08 & 0.31 & 0.57 \\
\hline & 21 & -1.21 & -1.17 & -1.12 & -1.05 & -0.97 & -0.87 & -0.75 & -0.61 & -0.46 & -0.28 & -0.07 & 0.16 & 0.41 \\
\hline \multirow[t]{7}{*}{$\mathrm{L}$} & 5 & 0.12 & 0.14 & 0.16 & 0.19 & 0.22 & 0.26 & 0.31 & 0.37 & 0.44 & 0.51 & 0.6 & 0.69 & 0.8 \\
\hline & 8 & -0.15 & -0.14 & -0.11 & -0.09 & -0.05 & -0.01 & 0.04 & 0.1 & 0.16 & 0.24 & 0.32 & 0.42 & 0.53 \\
\hline & 11 & -0.28 & -0.26 & -0.24 & -0.21 & -0.18 & -0.13 & -0.08 & -0.03 & 0.04 & 0.11 & 0.2 & 0.3 & 0.40 \\
\hline & 13 & -0.33 & -0.31 & -0.29 & -0.26 & -0.23 & -0.18 & -0.14 & -0.08 & -0.01 & 0.06 & 0.15 & 0.24 & 0.35 \\
\hline & 16 & -0.38 & -0.36 & -0.34 & -0.31 & -0.28 & -0.24 & -0.19 & -0.13 & -0.06 & 0.01 & 0.1 & 0.19 & 0.3 \\
\hline & 18 & -0.41 & -0.39 & -0.37 & -0.34 & -0.3 & -0.26 & -0.21 & -0.16 & -0.09 & -0.01 & 0.07 & 0.17 & 0.27 \\
\hline & 21 & -0.44 & -0.42 & -0.4 & -0.37 & -0.33 & -0.29 & -0.24 & -0.19 & -0.12 & -.04 & 0.04 & 0.14 & 0.25 \\
\hline \multirow[t]{7}{*}{ A } & 5 & 0.09 & 0.11 & 0.14 & 0.17 & 0.21 & 0.25 & 0.31 & 0.37 & 0.44 & 0.52 & 0.61 & 0.72 & 0.84 \\
\hline & 8 & -0.12 & -0.1 & -0.07 & -0.04 & -0 & 0.04 & 0.09 & 0.16 & 0.23 & 0.31 & 0.4 & 0.51 & 0.63 \\
\hline & 11 & -0.26 & -0.24 & -0.22 & -0.18 & -0.15 & -0.1 & -0.05 & 0.01 & 0.09 & 0.17 & 0.26 & 0.37 & 0.48 \\
\hline & 13 & $0-0.33$ & -.31 & -0.29 & -0.26 & -0.22 & -0.18 & -0.12 & -0.06 & 0.01 & 0.09 & 0.19 & 0.29 & 0.41 \\
\hline & 16 & -0.43 & -0.41 & -0.38 & -0.35 & -0.31 & -0.27 & -.22 & -0.15 & -0.08 & - & 0.09 & 0.2 & 0.31 \\
\hline & 18 & -0.48 & -0.46 & -0.44 & -0.41 & -0.37 & -0.32 & -0.27 & -0.21 & -0.13 & -0.05 & 0.04 & 0.14 & 0.26 \\
\hline & 21 & -0.55 & -0.53 & -0.51 & -0.47 & -0.44 & -0.39 & -0.34 & -0.28 & -0.2 & -0.12 & 0.03 & 0.08 & 0.19 \\
\hline \multirow[t]{7}{*}{ M } & 5 & -.56 & -0.43 & -0.29 & -0.13 & 0.05 & 0.24 & 0.45 & 0.68 & 0.92 & 1.18 & 1.45 & 1.75 & 2.06 \\
\hline & 8 & -0.8 & -0.68 & -0.53 & -0.37 & -0.2 & -0.01 & 0.2 & 0.43 & 0.67 & 0.93 & 1.21 & 1.5 & 1.81 \\
\hline & 11 & -1 & -0.88 & -0.73 & -0.58 & -0.4 & -0.21 & - & 0.23 & 0.47 & 0.73 & 1.01 & 1.3 & 1.61 \\
\hline & 13 & -1.12 & -1 & -0.85 & -0.69 & -0.52 & -0.33 & -0.12 & 0.11 & 0.35 & 0.61 & 0.89 & 1.18 & 1.49 \\
\hline & 16 & -1.29 & -1.16 & -1.02 & -0.86 & -0.68 & -0.49 & -0.28 & -0.05 & 0.19 & 0.45 & 0.72 & 1.02 & 1.33 \\
\hline & 18 & -1.39 & -1.26 & -1.12 & -0.96 & -0.78 & -0.59 & -0.38 & -0.15 & 0.09 & 0.35 & 0.62 & 0.92 & 1.23 \\
\hline & 21 & -1.53 & -1.4 & -1.26 & -1.1 & -0.92 & -0.73 & -0.52 & -0.29 & -0.05 & 0.21 & 0.48 & 0.78 & 1.09 \\
\hline $\mathrm{O}$ & & -0.13 & -0.11 & -0.09 & -0.08 & -0.06 & -0.03 & -0.01 & 0.02 & 0.06 & 0.1 & 0.15 & 0.23 & 0.37 \\
\hline
\end{tabular}

MoCA, Montreal Cognitive Assessment; VS, visuo-spatial; EF, executive functioning; L, language; A, attention; M, memory; O, orientation; Total: adjusted score $=$ raw score $+.000008 *\left[\left(\mathrm{age}^{3}\right)-297697.184801\right]-3.331407 *[\ln ($ education$)-2.325648]$; VS: adjusted score $=$ raw score $+.000155 *\left[\left(\mathrm{age}^{2}\right)-4168.682243\right]-0.645622 *[\ln ($ education $)-2.322051]$; EF: adjusted score $=$ raw score $+.000002 *\left[\left(\right.\right.$ age $\left.\left.^{3}\right)-290195.728972\right]-$ $.996668 *\left[\ln \left(\right.\right.$ education)-2.322051]; $\quad \mathrm{L}: \quad$ adjusted $\quad$ score $=$ raw $\quad$ score $+.00000083757 *\left[\left(\right.\right.$ age $\left.\left.^{3}\right)-\quad 290195.728972\right]+3.645727 *\left[\left(1 / e^{2}\right.\right.$ ducation)-0.110560]; A: adjusted score $=$ raw score $+.00000091089 *\left[\left(\right.\right.$ age $\left.\left.^{3}\right)-290195.728972\right]-0.448568 *[\ln ($ education)-2.322051]; M: adjusted score $=$ raw score $+.000335 *\left[\left(\mathrm{age}^{2}\right)-4168.682243\right]-0.413262 *[\operatorname{sqrt}($ education $)-3.276794] ;$ O: adjusted score $=$ raw score-0.191626*[ln $(100$ age)-3.515369]. Significant decimals of adjustment factors are displayed. Adjustment factors have been extracted from the aforementioned formula and do not always reflect empirical co-occurrences 
Table 4 Equivalent Scores for MoCA total and sub-test adjusted scores

\begin{tabular}{|c|c|c|c|c|c|c|c|}
\hline & \multirow[t]{2}{*}{ oTL } & \multirow[t]{2}{*}{ iTL } & \multicolumn{5}{|c|}{ Equivalent Scores } \\
\hline & & & 0 & 1 & 2 & 3 & 4 \\
\hline MoCA & 18.58 & 19.48 & $\leq 18.58$ & $18.59-20.69$ & $20.7-22.56$ & $22.57-24.52$ & $\geq 24.53$ \\
\hline MoCA-VS & 1.36 & 1.74 & $\leq 1.36$ & $1.37-2.03$ & $2.04-2.64$ & $2.65-3.22$ & $\geq 3.23$ \\
\hline MoCA-EF & 1.07 & 1.46 & $\leq 1.07$ & $1.08-1.87$ & $1.88-2.45$ & $2.46-3.07$ & $\geq 3.08$ \\
\hline MoCA-L & 2.98 & 3.44 & $\leq 2.98$ & $2.99-3.71$ & $3.72-4.15$ & $4.16-4.71$ & $\geq 4.72$ \\
\hline MoCA-A & 3.44 & 3.79 & $\leq 3.44$ & $3.45-4.5$ & $4.51-5.09$ & $5.1-5.66$ & $\geq 5.67$ \\
\hline MoCA-M* & - & 0.11 & - & $\leq 0.45$ & $.46-1.28$ & $1.29-2.29$ & $\geq 2.3$ \\
\hline MoCA-O & 4.92 & 4.97 & $\leq 4.92$ & $4.93-5.84$ & $5.85-5.93$ & $5.94-5.96$ & $\geq 5.97$ \\
\hline MoCA-AES & 1.83 & 2.33 & $\leq 1.83$ & - & - & - & - \\
\hline
\end{tabular}

MoCA, Montreal Cognitive Assessment; VS, visuo-spatial; EF, executive functioning; L, language; A, attention; M, memory; O, orientation; oTL, outer tolerance limit; iTL, inner tolerance limit; AES, Average Equivalent Score. *It is not possible to classify an adjusted score on the MoCA-M as ES $=0$. AESs are calculated by averaging ESs of each sub-test to provide a standardized across-domain global index observation corresponding to the first positive adjusted score was regarded as an empirical iTL (yielding a $p>0.99$ that $95 \%$ of the population performs above it). No adjusted score was thus classified as $\mathrm{ES}=0$.

AESs proved to be independent from sex $(t(533)=1.8$; $p=0.073)$, age $(r(535)=0.07 ; p=0.119)$ and education $(r(535)=0.03 ; p=0.44)$.

Weak agreement $(0.17 \leq k \leq 0.57)$ [42] was detected between the present and both

Conti et al.'s [9] and Santangelo et al.'s [10] ES classifications (see Table 5). More specifically, ESs allotments here reported proved to be more conservative than those of Santangelo et al.'s [10] with regard to MoCA-total, -VS, -EF and $-\mathrm{A}$, whereas less conservative with regard to $-\mathrm{L}$ and $-\mathrm{O}$ and Conti et al.'s [9] total.

As regards item-level analyses, the MoCA proved to be internally consistent (Cronbach's $\alpha=0.81$ ).

A mono-component factor (15.9\% of variance explained) structure was selected from PCA, with the majority of items highly loading $(0.3 \leq r \leq 0.55)$, except for $N=8$ items (CDT contour, digit span backward, lion and camel naming and all MoCA-O items except for year; $.02 \leq r \leq 0.26$ ).

Item difficulty and discrimination values are displayed in Table 6. The most difficult items proved to be the threedimension cube copy, CDT hands, repetition of the second sentence, phonemic fluency, the second verbal abstraction item and DR items. The least difficult ones were CDT contour, lion-naming, the letter detection task and month, place and city items of MoCA-O. TMT, repetition of the first sentence, DR items and year and city of MoCA-O proved to be the most effective in discriminating between different levels of ability, whilst those with the lowest values of discrimination were place of MoCA-O and the letter detection task.

\section{Discussion}

The present work provides Italian practitioners with updated, region-specific normative data for the MoCA, as well as with IRT-based, item-level information that may allow a more flexible and informative use of this screening instrument.

Although norms for the Italian MoCA have been provided in previous studies $[9,10]$, recent changes in demographic composition and socio-cultural features of Italian population motivated the normative branch of this study. Moreover, the present sample covers wider ranges of age and education and is larger ( $N=579$; age: $21-96$; education: $1-25)$ when compared to previous normative studies - Conti et al. [9]: $N=225$; age: 60-80; education: 5-23; Santangelo et al. [10]: $N=415$; age: $21-95$; education: $1-21$. Norms here reported are thus likely to be more representative and generalizable as far as sample size and coverage of anagraphic-demographic variables are concerned.

Moreover, the oTL for MoCA-M had not been provided by Santangelo et al. [10] because it corresponded to a negative adjusted score. Nonetheless, despite this finding having been replicated also in the present study, an empirical iTL for MoCA-M has been with provided, along with ESs thresholds (which, however, did not correspond to negative adjusted scores). Although caution is needed when interpreting this iTL, its practical use is quite intuitive. For instance, only for young and highly educated individuals a raw score of 1 would be classified as below the aforementioned iTL. Thereupon, practitioners would not be allowed to judge that a score below the MoCA-M iTL falls in the worst $5 \%$ of the population, although it would be possible to say that $99 \%$ of healthy individuals perform above it. 
Table 5 Comparison between Equivalent Scores classifications

\begin{tabular}{|c|c|c|c|c|c|c|}
\hline \multirow{3}{*}{$\begin{array}{l}\text { Previous classifications } \\
\text { Santangelo } \text { et al. } \\
\text { Total }\end{array}$} & \multicolumn{6}{|c|}{ Present classification } \\
\hline & & $\mathbf{0}$ & 1 & 2 & 3 & 4 \\
\hline & 0 & 5 & 0 & 0 & 0 & 0 \\
\hline & 1 & 8 & 2 & 0 & 0 & 0 \\
\hline & 2 & 8 & 22 & 7 & 0 & 0 \\
\hline & 3 & 0 & 22 & 60 & 5 & 0 \\
\hline & 4 & 0 & 0 & 27 & 126 & 287 \\
\hline VS & $\overline{\mathbf{0}}$ & 4 & 0 & 0 & 0 & 0 \\
\hline & 1 & 10 & 2 & 0 & 0 & 0 \\
\hline & 2 & 3 & 33 & 3 & 0 & 0 \\
\hline & 3 & 0 & 11 & 55 & 3 & 0 \\
\hline & 4 & 0 & 0 & 28 & 115 & 268 \\
\hline EF & $\mathbf{0}$ & 2 & 0 & 0 & 0 & 0 \\
\hline & 1 & 13 & 7 & 0 & 0 & 0 \\
\hline & 2 & 3 & 31 & 7 & 0 & 0 \\
\hline & 3 & 0 & 6 & 69 & 16 & 1 \\
\hline & 4 & 0 & 0 & 9 & 105 & 266 \\
\hline $\mathbf{A}$ & $\mathbf{0}$ & 5 & 0 & 0 & 0 & 0 \\
\hline & 1 & 11 & 11 & 0 & 0 & 0 \\
\hline & 2 & 3 & 20 & 14 & 1 & 0 \\
\hline & 3 & 0 & 12 & 58 & 34 & 8 \\
\hline & 4 & 0 & 0 & 12 & 86 & 260 \\
\hline $\mathbf{O}$ & $\mathbf{0}$ & 21 & 15 & 0 & 0 & 0 \\
\hline & 1 & 0 & 29 & 44 & 6 & 0 \\
\hline & 2 & 0 & 0 & 13 & 21 & 3 \\
\hline & 3 & 0 & 0 & 31 & 75 & 23 \\
\hline & 4 & 0 & 0 & 13 & 30 & 211 \\
\hline
\end{tabular}

Conti et al.

\begin{tabular}{cc|c|c|c|c|c|} 
Total & $\mathbf{0}$ & 15 & 7 & 2 & 2 & 0 \\
& $\mathbf{1}$ & 6 & 30 & 21 & 3 & 2 \\
& $\mathbf{2}$ & 0 & 9 & 61 & 39 & 9 \\
& $\mathbf{3}$ & 0 & 0 & 10 & 69 & 45 \\
& $\mathbf{4}$ & 0 & 0 & 0 & 18 & 231 \\
\hline
\end{tabular}

MoCA, Montreal Cognitive Assessment; VS, visuo-spatial; EF, executive functioning; A, attention; M, memory; O, orientation. Diagonal co-occurrences index inter-rater agreements; extra-diagonal co-occurrences index disagreements (below the diagonal: the present classification is more conservative; above the diagonal: the present classification is less conservative). Language sub-test could not be compared due to different ranges

With respect to anagraphic-demographic predictors, MoCA-O scores proved not to be influenced by education in the present study. This finding diverges from previous ones regarding not only the MoCA [10], but also other cognitive screening tests $[43,44]$. Similarly, although males were found as performing better than females on MoCA-A and -VS, when sex was tested individually, no such differences have been yielded from models additionally accounting for age and education, contrarily to Santangelo et al.'s [10] study. This finding was also true for MoCA-L, although it has not been previously reported [10]. This discrepancy may be attributed to age/education voiding sex differences in this larger sample, and it is in line with inconsistent findings in concerning literature [45].

Along with the above inconsistencies regarding anagraphic-demographic variables, the fact that the present
Table 6 Item difficulty and discrimination for the MoCA

\begin{tabular}{|c|c|c|}
\hline Item & Difficulty & Discrimination \\
\hline TMT & -1.347 & $1.527^{\mathrm{c}}$ \\
\hline Cube & -0.892 & 1.247 \\
\hline CDT-C & $-5.416^{\mathrm{b}}$ & 0.697 \\
\hline CDT-N & -1.505 & 0.64 \\
\hline CDT-H & -0.946 & 1.331 \\
\hline Lion & $-12^{\mathrm{b}}$ & 0.44 \\
\hline Rhino & -1.86 & 1.373 \\
\hline Camel & $-3.803^{\mathrm{a}}$ & 1.333 \\
\hline FDS & $-2.611^{\mathrm{a}}$ & 0.827 \\
\hline BDS & $-3.19^{\mathrm{a}}$ & 0.644 \\
\hline A & $-11.22^{b}$ & 0.221 \\
\hline 93 & $-3.299^{\mathrm{a}}$ & 1.126 \\
\hline 86 & -1.778 & 0.812 \\
\hline 79 & $-2.243^{\mathrm{a}}$ & 0.904 \\
\hline 72 & -1.856 & 1.108 \\
\hline 65 & $-2.037^{\mathrm{a}}$ & 0.934 \\
\hline Rep. 1 & $-2.676^{\mathrm{a}}$ & $1.691^{\mathrm{c}}$ \\
\hline Rep. 2 & -0.961 & 0.862 \\
\hline Flu & -0.862 & 1.1 \\
\hline Abst. 1 & -1.55 & $1.606^{\mathrm{c}}$ \\
\hline Abst. 2 & -0.488 & 0.724 \\
\hline DR 1 & 0.56 & $1.663^{\mathrm{c}}$ \\
\hline DR 2 & 0.064 & $1.919^{\mathrm{d}}$ \\
\hline DR 3 & -0.05 & $1.446^{\mathrm{a}}$ \\
\hline DR 4 & 0.325 & $1.962^{\mathrm{d}}$ \\
\hline DR 5 & -0.162 & $1.863^{\mathrm{d}}$ \\
\hline Date & $-2.993^{\mathrm{a}}$ & 1.022 \\
\hline Month & $-5.984^{b}$ & 0.772 \\
\hline Year & $-3.347^{\mathrm{a}}$ & $1.512^{\mathrm{c}}$ \\
\hline Day & -3.68 & 1.22 \\
\hline Place & $-124^{b}$ & 0.034 \\
\hline City & $-4.157^{b}$ & $1.732^{\mathrm{d}}$ \\
\hline
\end{tabular}

MoCA, Montreal Cognitive Assessment; TMT, Trail Making Test; CDT, Clock Drawing Test; -C, contour; -N, numbers; -H, hands; FDS, forward digit span; BDS, backward digit span; A, letter detection task; Rep., sentence repetition; Flu., phonemic fluency; Abst., abstraction task; DR, delayed recall. Higher values correspond to higher difficulty and discriminative capability of items. ${ }^{a}$ low difficulty; ${ }^{b}$ very low difficulty; ${ }^{c}$ high discrimination; (Hambleton et al. [30]) ${ }^{d}$ very high discrimination (Baker and Kim [31]). Very low difficulty items $(\leq-4)$ were identified by doubling the "cut-off" value for "very easy" items $(\leq-2)$ established by Hambleton et al. [30]

cut-off thresholds happened to systematically diverge from those of Conti et al. [9] and Santangelo et al. [10] is suggestive of relevant inter-regional differences that should be taken into consideration by Northern Italian practitioners [46]. It is noteworthy that this last aspect has been recently addressed in Italy with respect to the Mini-Mental State Examination [47], for whom region-specific norms have been recently provided for Southern Italian individuals. 
Major contributions to an adaptive interpretation [48, 49] of the Italian MoCA also come from single-item-level analyses, which indicate the need to pay particular attention to highly discriminative items when specificity has to be favored, and to highly difficult ones when sensitivity does. Of relevance, despite cultural/language differences [24], the present findings are in line with previous ones from eastern countries with regard to the high discriminative capability of MoCA-EF and -M items $[18,19]$.

This work has a main limitation that needs consideration: a different cognitive screening test was not administered since it was out of the present aims to assess concurrent/convergent validity of the MoCA. However, due to the lack of such data, it is not possible to rule out sub-clinical cognitive deficits in participants. It is also noteworthy that item- and sub-test-level analyses were performed on a smaller sample (535 out of 579 participants) due to completely-at-random missing values [50].

In conclusion, the present study and its results favor a more informative and flexible use, scoring and interpretation of the Italian MoCA by providing updated and region-specific normative data at the sub-test level, also comprising a proxy cut-off for MoCA-M scores; moreover, novel information on sensitivity and discriminative capability of single Italian MoCA items have been provided.

Acknowledgements We are grateful to Dr. Ziad Nasreddine for providing us with his permission to use the MoCA for experimental scopes.

Funding Open access funding provided by Università degli Studi di Milano - Bicocca within the CRUI-CARE Agreement. This study did not receive any specific funding.

Data availability Data collected and analyzed during the present study are available on the Open Science Framework (OSF) repository (https://osf.io/cykbe/).

\section{Declarations}

Conflict of interests The authors declare no known conflicts of interests.

Ethical approval This study was conducted in accordance with the Declaration of Helsinki. All of these studies were approved by the Research Evaluation Committee of the Department of Psychology of University of Milano-Bicocca on behalf of the Ethical Committee of the same Institution.

Informed consent Participants provided informed consent and signed a data treatment disclaimer for research purposes.

Open Access This article is licensed under a Creative Commons Attribution 4.0 International License, which permits use, sharing, adaptation, distribution and reproduction in any medium or format, as long as you give appropriate credit to the original author(s) and the source, provide a link to the Creative Commons licence, and indicate if changes were made. The images or other third party material in this article are included in the article's Creative Commons licence, unless indicated otherwise in a credit line to the material. If material is not included in the article's Creative Commons licence and your intended use is not permitted by statutory regulation or exceeds the permitted use, you will need to obtain permission directly from the copyright holder. To view a copy of this licence, visit http://creativecommons.org/licenses/by/4.0/.

\section{References}

1. Mitrushina M (2009) Cognitive screening methods. In: Grant I, Adams KM (eds) Neuropsychological Assessment of Neuropsychiatric and Neuromedical Disorders. Oxford University Press, pp 101-126

2. Tsoi KK, Chan JY, Hirai HW et al (2015) Cognitive tests to detect dementia: a systematic review and meta-analysis. JAMA Intern Med 175:1450-1458

3. Trzepacz PT, Hochstetler H, Wang S et al (2015) Relationship between the Montreal Cognitive Assessment and Mini-mental State Examination for assessment of mild cognitive impairment in older adults. BMC Geriatr 15:1-9

4. Trevethan R (2017) Sensitivity, specificity, and predictive values: foundations, pliabilities, and pitfalls in research and practice. Front Public Health 5:307

5. Willmes K (2010) The methodological and statistical foundations of neuropsychological assessment. In: Gurd J, Kischka U, Marshall J (eds) The Handbook of Clinical Neuropsychology, 2nd edn, Oxford University Press, New York, pp 28-49

6. Bilder RM, Reise SP (2019) Neuropsychological tests of the future: how do we get there from here? Clin Neuropsychol $33: 220-245$

7. Nasreddine ZS, Phillips NA, Bédirian V et al (2005) The Montreal Cognitive Assessment, MoCA: a brief screening tool for mild cognitive impairment. J Am Geriatr Soc 53:695-699

8. Julayanont P, Nasreddine ZS (2017) Montreal Cognitive Assessment (MoCA): concept and clinical review. In: Larner AJ (ed) Cognitive Screening Instruments. Springer, pp 139-195

9. Conti S, Bonazzi S, Laiacona $\mathrm{M}$ et al (2015) Montreal Cognitive Assessment (MoCA)-Italian version: regression based norms and equivalent scores. Neurol Sci 36:209-214

10. Santangelo G, Siciliano M, Pedone R et al (2015) Normative data for the Montreal Cognitive Assessment in an Italian population sample. Neurol Sci 36:585-591

11. Bosco A, Spano G, Caffò AO et al (2017) Italians do it worse. Montreal Cognitive Assessment (MoCA) optimal cut-off scores for people with probable Alzheimer's disease and with probable cognitive impairment. Aging Clin Exp Res 29:1113-1120

12. Siciliano M, Chiorri C, Passaniti C et al (2019) Comparison of alternate and original forms of the Montreal Cognitive Assessment (MoCA): an Italian normative study. Neurol Sci 40:691-702

13. Moafmashhadi P, Koski L (2013) Limitations for interpreting failure on individual subtests of the Montreal Cognitive Assessment. J Geriatr Psychiatry Neurol 26:19-28

14. Cecato JF, Martinelli JE, Izbicki R et al (2016) A subtest analysis of the Montreal cognitive assessment (MoCA): which subtests can best discriminate between healthy controls, mild cognitive impairment and Alzheimer's disease? Int Psychogeriatr 28:825

15. Lord FM (1971) The self-scoring flexilevel test 1. J Educ Meas $8: 147-151$

16. Thomas ML (2011) The value of item response theory in clinical assessment: a review. Assessment 18:291-307

17. Baylor C, Hula W, Donovan NJ, et al. (2011) An introduction to item response theory and Rasch models for speech-language pathologists. Am J Speech Lang Pathol 20:243-259 
18. Tsai CF, Lee WJ, Wang SJ et al (2012) Psychometrics of the Montreal Cognitive Assessment (MoCA) and its subscales: validation of the Taiwanese version of the MoCA and an item response theory analysis. Int Psychogeriatr 24:651

19. Zheng L, Teng EL, Varma R et al (2012) Chinese-language Montreal Cognitive Assessment for Cantonese or Mandarin speakers: age, education, and gender effects. Int J Alzheimer's Dis 2012:10

20. Freitas S, Prieto G, Simões MR et al (2014) Psychometric properties of the Montreal Cognitive Assessment (MoCA): an analysis using the Rasch model. Clin Neuropsychol 28:65-83

21. Luo H, Andersson B, Tang JY et al (2020) Applying item response theory analysis to the Montreal Cognitive Assessment in a loweducation older population. Assessment 27:1416-1428

22. Pedraza O, Mungas D (2008) Measurement in cross-cultural neuropsychology. Neuropsychol Rev 18:184-193

23. Manly JJ (2008) Critical issues in cultural neuropsychology: Profit from diversity. Neuropsychol Rev 18:179

24. Uysal-Bozkir Ö, Parlevliet JL, de Rooij SE (2013) Insufficient cross-cultural adaptations and psychometric properties for many translated health assessment scales: a systematic review. J Clin Epidemiol 66:608-618

25. Siciliano M, Chiorri C, Battini V et al (2019) Regressionbased normative data and equivalent scores for Trail Making Test (TMT): an updated Italian normative study. Neurol Sci 40:469-477

26. Pirani A, Tulipani C, Neri M (2006) Montreal Cognitive Assessment, Italian version

27. Ghasemi A, Zahediasl S (2012) Normality tests for statistical analysis: a guide for non-statisticians. Int J Endocrinol Metab 10:486

28. Kim HY (2013) Statistical notes for clinical researchers: assessing normal distribution (2) using skewness and kurtosis. Restorative Dentistry Endodontics 38:52-54

29. Rizopoulos D (2006) ltm: An R package for latent variable modelling and item response theory analyses. J Stat Softw 17:1-25

30. Hambleton RK, Swaminathan H, Rogers HJ (1991) Fundamentals of item response theory. Sage Publications, California, pp 7-31

31. Baker FB, Kim SH (2017) The basics of item response theory using R. Springer, New York, pp 17-34

32. Spinnler H, Tognoni G (1987) Standardizzazione e taratura italiana di test neuropsicologici. Ital J Neurol Sci 6:1-120

33. Capitani E, Laiacona M (2017) Outer and inner tolerance limits: their usefulness for the construction of norms and the standardization of neuropsychological tests. Clin Neuropsychol 31:1219-1230

34. Capitani E, Laiacona M (1997) Composite neuropsychological batteries and demographic correction: standardization based on equivalent scores, with a review of published data. J Clin Exp Neuropsychol 19:795-809

35. Rothstein HR, Borenstein M, Cohen J et al (1990) Statistical power analysis for multiple regression/correlation: a computer program. Educ Psychol Measur 50:819-830

36. Champely S (2020) pwr: Basic functions for power analysis $(\mathrm{R}$ package version 1.3-0) (Computer software). The Comprehensive R Archive Network. https://CRAN.R-project.org/package=pwr
37. Brugnolo A, De Carli F, Accardo J et al (2016) An updated Italian normative dataset for the Stroop color word test (SCWT). Neurol Sci 37:365-372

38. Tremolizzo L, Lizio A, Santangelo G, et al. ALS-CBS Italian Study Group (2020) ALS Cognitive Behavioral Screen (ALSCBS): normative values for the Italian population and clinical usability. Neurol Sci 41:835-841

39. IBM Corp (2020) IBM SPSS Statistics for Windows, Version 27.0. Armonk, NY: IBM Corp

40. R Core Team (2019) R: A Language and environment for statistical computing. (Version 3.6.3) [Computer software]. Retrieved from https://cran.r-project.org

41. Aiello EN, Depaoli EG (2021) Norms and standardizations in neuropsychology via equivalent scores: software solutions and practical guides. Neurol Sci. https://doi.org/10.1007/ s10072-021-05374-0

42. McHugh ML (2012) Interrater reliability: the kappa statistic. Biochemia Medica 22:276-282

43. Siciliano M, Raimo S, Tufano D et al (2016) The Addenbrooke's Cognitive Examination Revised (ACE-R) and its sub-scores: normative values in an Italian population sample. Neurol Sci 37:385-392

44. Pigliautile M, Chiesi F, Stablum F et al (2019) Italian version and normative data of Addenbrooke's Cognitive Examination III. Int Psychogeriatr 31:241-249

45. Hirnstein M, Hugdahl K, Hausmann M (2019) Cognitive sex differences and hemispheric asymmetry: a critical review of 40 years of research. Laterality: Asymmetries of Body. Brain Cogn 24:204-252

46. Bush SS (2010) Determining whether or when to adopt new versions of psychological and neuropsychological tests: ethical and professional considerations. Clin Neuropsychol 24:7-16

47. Mazzi MC, Iavarone A, Russo G et al (2020) Mini-Mental State Examination: new normative values on subjects in Southern Italy. Aging Clin Exp Res 32:699-702

48. Hannay HJ, Lezak MD (2004) The neuropsychological examination: interpretation. In: Lezak MD, Howieson DB, Loring DW (eds) Neuropsychological assessment. Oxford University Press, New York, pp 133-155

49. Kolakowsky-Hayner SA, Caplan B (2011) Qualitative Neuropsychological Assessment. In: Kreutzer JS, DeLuca J, Caplan B (eds) Encyclopedia of Clinical Neuropsychology. Springer, New York, pp 2098-2099

50. Masconi KL, Matsha TE, Echouffo-Tcheugui JB et al (2015) Reporting and handling of missing data in predictive research for prevalent undiagnosed type 2 diabetes mellitus: a systematic review. EPMA J 6:1-11

Publisher's Note Springer Nature remains neutral with regard to jurisdictional claims in published maps and institutional affiliations. 